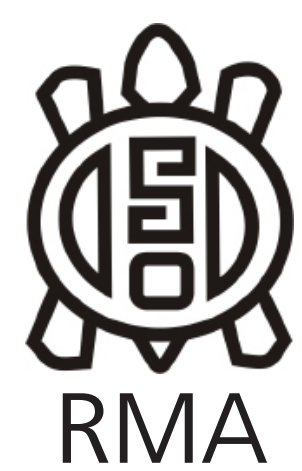

Antropología Biológica

\title{
Caracterización bioarqueológica e isotópica de restos óseos humanos de un entierro del sitio tardío El Molino (Depto. Belén, Catamarca)
}

\author{
Bioarchaeological and isotopic characterization of human remains \\ from a burial of the Late Period site El Molino (Belén Department, \\ Catamarca Province) \\ María Emilia Iucci ${ }^{1,2}$, Virginia A. Cobos ${ }^{1,2}$, \\ Bruno Moscardi ${ }^{1}$ y S. Ivan Perez ${ }^{1,2}$
}

\begin{abstract}
1 Facultad de Ciencias Naturales y Museo, Universidad Nacional de La Plata, La Plata, Argentina. E-mail: emiliaiucci@fcnym.unlp.edu.ar, virginiacobos@gmail.com, bruno.f.moscardi@gmail.com e ivanperezmorea@gmail.com

${ }^{2}$ Consejo Nacional de Investigaciones Científicas y Técnicas (CONICET), Buenos Aires, Argentina.
\end{abstract}

\begin{abstract}
Resumen
Se analizan los restos esqueletales hallados en una estructura excavada en el sitio El Molino, un poblado prehispánico tardío localizado en el Valle de Hualfín. El hallazgo se realizó en un sector cerrado por una pirca, en el interior de un recinto de características habitacionales, y constituye uno de los escasos ejemplos de restos humanos de un individuo adulto adecuadamente contextualizado y registrado de la región. Los estudios bioarqueológicos permitieron determinar que se trata de un individuo femenino de entre 45 y 50 años, con presencia de alteraciones degenerativas de las articulaciones, desgaste y patologías dentales, frecuentes en grupos humanos agricultores. El análisis isotópico resultó consistente con una dieta basada en camélidos de regiones bajas y recursos vegetales procesados, posiblemente maíz. La realización de un fechado permite ubicar al entierro en el siglo XV, de manera análoga a la mayoría de fechados tardíos obtenidos en el valle.
\end{abstract}

Palabras claves: Valle de Hualfín; Perfil Paleodemográfico; Paleopatología; Datos Isotópicos; Dieta Andina

\begin{abstract}
The skeletal remains found in a structure excavated at the El Molino site, a Late Period prehispanic settlement located in the Hualfin Valley, are analyzed. The finding was made in a sector closed by a stone wall -pirca-, within a structure of dwelling characteristics, and constitutes one of the few examples of human remains of an adult individual properly contextualized and registered in the region. Bioarchaeological studies allowed us to determine that it is a female individual between 45 and 50 years old, with the presence of degenerative alterations of the joints, wear and dental pathologies, frequents in farmer human groups. The isotopic analysis was consistent with a diet based on lowland camelids and processed plant resources, possibly corn. The burial was dated to the 15th century, and is coherent with the majority of the late dates obtained in the valley.
\end{abstract}

Key words: Hualfín Valley, Paleodemographic Structure, Paleopathology, Isotopic Data, Andean Diet

Los contextos funerarios del Valle de Hualfín son bien conocidos a través de la importante cantidad de tumbas excavadas en la década de 1920 por W. Weisser y F. Wolters, en el marco de las expediciones financiadas por B. Muñiz Barreto al Noroeste Argentino, a partir de las que se conformó la Colección Benjamín Muñiz Barreto del Museo de La Plata con 12.000 piezas arqueológicas. En el soporte documental que la acompaña se registra la localización y características de los sepulcros y piezas asociadas, y el número de individuos inhumados con una estimación etaria aproximada efectuada en el campo durante las excavaciones. Los restos esqueletales no fueron recuperados, y los detalles sobre las características biológicas de quienes fueron inhumados no se conocen
(Balesta y Zagorodny, 2000; Sempé, Salceda y Maffia, 2005). A partir de la década de 1950 se realizaron otros trabajos arqueológicos en el valle (González, 1955; Sempé, 1981; Onaha, Tobisch, Padula, Drube y Vasallo, 2002; Wynveldt, 2009, entre otros), en el marco de los que, ocasionalmente, se ubicaron sepulcros infantiles individuales o múltiples (Sempé, Méndez y Salceda, 19951996; Wynveldt, 2009; Balesta y García Mancuso, 2010). Entre estos entierros sólo se registraron dos casos de adultos, uno en el sitio Loma de los Antiguos (Wynveldt, 2009), y el otro en la mesada de Carrizal (Onaha et al., 2002). Adicionalmente, en la zona se conocen numerosos entierros exhumados en contextos de rescates arqueológicos, que han permanecido sin ser estudiados, 
con la excepción de tres sepulcros recuperados en las tareas de estudio de impacto para el trazado de la Ruta Nacional No 40 (Palamarczuk y Ratto, 2015). Por otra parte, en la localidad de Asampay se identificó una nueva área de entierros de mayor escala, relacionada con las ocupaciones humanas de mayor antigüedad conocidas en la zona (Lamenza et al., 2016).

En este trabajo se presentan los resultados del estudio bioarqueológico de un esqueleto recientemente recuperado en el sitio El Molino de la localidad actual de Puerta de Corral Quemado, que constituye uno de los escasos hallazgos correctamente contextualizado y documentado de un individuo adulto para los momentos prehispánicos tardíos.

\section{El entierro en el contexto del sitio El Molino}

El Molino se ubica sobre una lomada de unos $70 \mathrm{~m}$ de altura en la orilla sur del Río Corral Quemado (27 $13,823^{\prime}$ S y $66^{\circ} 56,643^{\prime}$ W, a 1932 msnm), y está formado por aproximadamente 110 estructuras construidas en piedra. W. Weiser (1924) excavó entierros en sus alrededores y realizó el plano del sitio (Figura 1). A. R. González excavó algunas estructuras en 1969 y realizó un fechado radiocarbónico que lo condujo a incluir al sitio en la fase Belén II de su secuencia cronológica, aunque esta asignación a los momentos tempranos de las ocupaciones Belén fue puesta en duda por él mismo (González y Cowgill, 1975). Más recientemente, se fecharon los restos esqueletales de un niño inhumado en una vasija que había sido hallado por González durante los trabajos de 1969 (García Mancuso e lucci, 2008). Esta nueva datación ubicó a El Molino, de manera análoga a otros sitios tardíos del valle, en el siglo XV (Wynveldt e lucci, 2013).

En 2016 se pudo dar inicio a una nueva etapa de trabajo arqueológico en el sitio. El individuo estudiado en el presente trabajo proviene de un sector delimitado en el recinto 34, una estructura de aproximadamente $5 x$ $5 \mathrm{~m}$, construida en cantos rodados, que entre 2016 y 2017 fue excavada por completo hasta llegar debajo del piso de ocupación, a los $80 \mathrm{~cm}$ de profundidad. El sector del entierro, en el ángulo sudoeste del recinto, estaba delimitado por una pirca oblicua a las paredes. El individuo se hallaba en posición decúbito lateral y estaba acompañado por un puco Belén (Figura 1). En el resto de la estructura se hallaron abundantes materiales en posición dispersa: fragmentos de cerámica Belén, Santa María y ordinaria, restos zooarqueológicos y macrobotánicos variados, artefactos líticos, un mortero de piedra y cuentas de aragonita.

Para su estudio, el esqueleto fue trasladado en 2017 a la ciudad de La Plata, con el permiso de la Dirección Provincial de Antropología de Catamarca. Luego de los análisis pertinentes en la División Antropología de la Facultad de Ciencias Naturales y Museo, en junio de 2018 fue devuelto a la provincia, y depositado en el Centro Turístico y Cultural de Puerta de Corral Quemado, donde se encuentran alojados algunos materiales arqueológicos recuperados en el sitio. Los resultados del presente estudio, junto con el lugar de depósito del esqueleto, fueron informados a la comunidad.

\section{Materiales y Métodos}

Se calculó el Número de Especímenes Óseos Identificados (NISP), el Número Mínimo de Elementos Óseos (NME) y el Número Mínimo de Individuos (NMI) (Lyman, 1994; Mengoni Goñalons, 1999), para confirmar la presencia de un único individuo en el entierro, así como estimar su grado de completitud (NME/Total de huesos en el esqueleto) e integridad (NME/NISP). Con este fin se dispusieron todos los elementos del entierro sobre una mesa de trabajo, se identificaron los huesos presentes y se contó cada unidad anatómica considerando lateralidad. Se evaluó el estado de conservación del individuo mediante una observación macroscópica detallada y se consideraron distintas variables tafonómicas: presencia de raíces, depósito de minerales, pérdida, fragmentación y descamación ósea, actividad de roedores y carnívoros, así como marcas de corte (Lyman, 1994; Mengoni Goñalons, 1999).

La edad biológica se estimó empleando las diez fases de edad de las modificaciones en la sínfisis púbica establecidas por Todd (1920; en White y Folkens, 2005,
Figura 1. a. Plano del sitio, con la estructura excavada señalada con un círculo. b. Disposición del área de entierro y del esqueleto inhumado en la estructura. c. Puco Belén hallado junto al esqueleto.

Figure 1. a. Site plan, with the excavated structure circled. $\mathbf{b}$. Disposition of the burial area and the skeleton buried in the structure. c. Belén bowl found next to the skeleton.
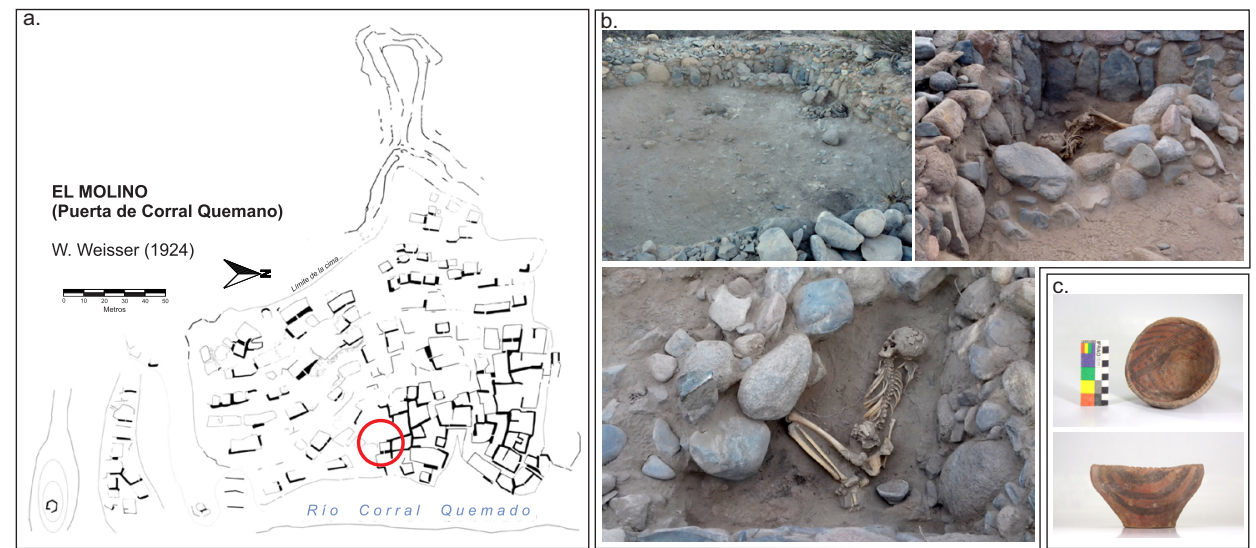
p. 376) y los cambios morfológicos en la superficie articular del íleon establecidos por Lovejoy y coautores (1985; en White y Folkens, 2005, p. 380). Se estimó el sexo considerando el arco ventral del pubis, la concavidad sub-púbica, el aspecto medial de la rama isquio-púbica, la escotadura ciática mayor, y el aspecto general de la proyección del pubis y de la fosa ilíaca (Ubelaker, 2008). Con este mismo fin, se observó la morfología del proceso mastoideo, la glabela, el arco supra-orbital, las órbitas y la mandíbula, como así también, se midió el diámetro perpendicular de la cabeza del fémur (Ubelaker, 2008).

Se estimó el desgaste dental, que se relaciona con la edad al momento de la muerte (aunque de manera muy variable) y con la dieta del individuo, mediante la medición del porcentaje de corona remanente usando el método de Smith (1984) para incisivos, caninos y premolares, y el de Scott (1979) para molares. A su vez se buscaron lesiones traumáticas y patológicas, y se observó el hueso frontal para determinar la presencia de deformación artificial del cráneo considerando los criterios de Dembo e Imbelloni (1938).

Finalmente, se extrajeron dos muestras de costilla para realizar un fechado radiocarbónico y el análisis de isótopos del carbono $\left(\delta^{13} \mathrm{C}\right)$ y nitrógeno $\left(\delta^{15} \mathrm{~N}\right)$. La calibración en años calendario del fechado obtenido se realizó con el programa CALIB Rev. 7.0.4, empleando la curva para el hemisferio sur SHCal13 (Hogg et al., 2013). Este programa se usó para determinar la similitud estadística con el fechado obtenido previamente. El proceso de extracción del colágeno para la realización de isótopos se llevó a cabo en las instalaciones de la División Antropología en el Edificio Anexo Laboratorios del Museo de la Facultad de Ciencias Naturales y Museo (Universidad Nacional de La Plata), según el procedimiento descrito por Gordón, Perez, Hajduk, Lezcano y Bernal (2018). Las determinaciones de los isótopos estables en el colágeno se realizaron en el laboratorio de la Washington State University. Los valores isotópicos de $\delta^{13} \mathrm{C}$ y $\delta^{15} \mathrm{~N}$ obtenidos fueron comparados con los de muestras previamente publicadas para humanos (Olivera y Yacobaccio, 1999; Killian Galván y Samec, 2012) y recursos de flora y fauna (Dantas, Figueroa, Laguens e Izeta, 2014; Killian Galván, Sanmartino, Castellano, Seldes y Marban, 2015; Grant, 2016; Samec, Pirola, Yacobaccio y Panarello, 2018) provenientes de distintas localidades del Noroeste Argentino (Tabla Suplementaria 1). Las muestras humanas fueron clasificadas según su región de origen, mientras que los recursos (particularmente los camélidos y las plantas (3) fueron divididos en "Altos" y "Bajos" según se ubicaran sobre o debajo de los 3.700 msnm, con el fin de explorar la variación isotópica altitudinal sugerida en trabajos previos (Killian Galván y Samec, 2012; Samec, Yacobaccio y Panarello, 2017; Tabla Suplementaria 2). Todos los valores isotópicos de muestras actuales fueron corregidos por el efecto Suess. Se generó un espacio isotópico que incluye los valores de humanos y de sus recursos con el programa MixSiar para $R$
(Stock y Semmens, 2016) y los valores de fraccionamiento isotópico informados por Gordón et al. (2018).

\section{Resultados y discusión}

El entierro cuenta de un único individuo humano (NMI= 1), con un grado de completitud para el post-cráneo del $61 \%$ (Figura 2a), mientras que el cráneo está completo pero muy fragmentado. El grado de integridad para el post-cráneo fue del $92 \%$. Si bien todos los elementos anatómicos se encuentran muy afectados por la acción de raíces, presentan un grado similar de preservación, de moderado a bueno, con excepción del neurocráneo y la cabeza de los fémures que están muy mal preservados. Sin embargo, estos últimos mantienen su tamaño y forma, lo cual permitió su utilización para la estimación de sexo del individuo. El grado de completitud dental es del 53\% (Figura 2b), con pérdidas observadas tanto pre-mortem como post-mortem.

Se determinó que el individuo recuperado es un adulto mayor de 45 a 50 años [(Fase 5 de Lovejoy et al. (1985) y IX de Todd (1920)] de sexo femenino (pubis proyectado y escotadura amplia, diámetro perpendicular de la cabeza del fémur $=42,5 \mathrm{~mm}$ ). En relación con el desgaste dental, pudo determinarse que los incisivos, caninos y premolares presentan un grado de desgaste moderado, que se corresponde con los estadios 5 - 6 de Smith (1984). Por otro lado, los molares presentan un grado de desgaste más heterogéneo, que varía de moderado a alto, entre los estadios 3 a 9 de Scott (1979).

Cabe destacar que, si bien no se identificaron lesiones traumáticas ni deformación artificial del cráneo, se observó la presencia de numerosas lesiones patológicas, probablemente de grado sistémico, principalmente en los miembros superiores e inferiores y en las vértebras (Figura 3). Asimismo, se identificó la presencia de abscesos (debajo del primer premolar mandibular izquierdo y derecho; Figura 2), aunque no se registraron caries, probablemente debido al elevado grado de desgaste dental.

La presencia de patologías dentales, como abscesos, son frecuentes en individuos pertenecientes a las sociedades agrícolas, probablemente como consecuencia de un consumo excesivo de hidratos de carbono (Larsen, 1987). En el mismo sentido, en estas sociedades es frecuente observar grados moderados a altos de desgaste, atribuibles al consumo de minerales desprendidos de artefactos de molienda junto con los alimentos (Onaha et al., 2002). Las características de las patologías postcraneales y dentales del individuo de El Molino se alinean con las inferidas para el individuo del sitio Mesada de Carrizal (Onaha et al., 2002). Los individuos de ambos sitios presentan señales de haber padecido osteoartritis $\mathrm{u}$ otras alteraciones degenerativas de las articulaciones en distintas zonas del esqueleto. En el caso del individuo de Mesada de Carrizal, Onaha y colaboradores (2002) 
a.

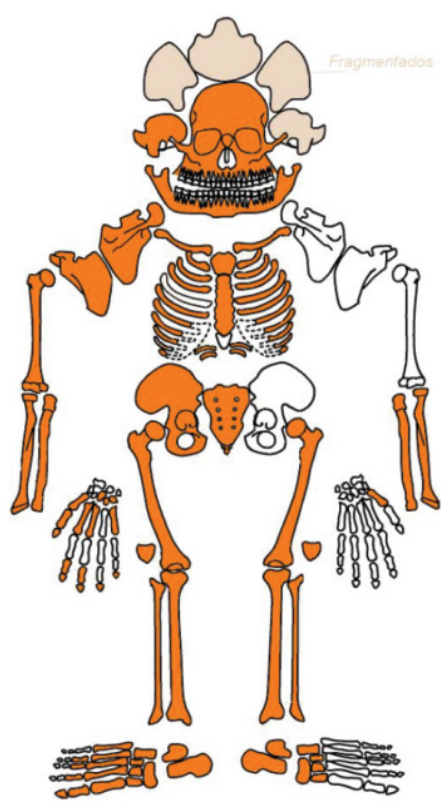

b.

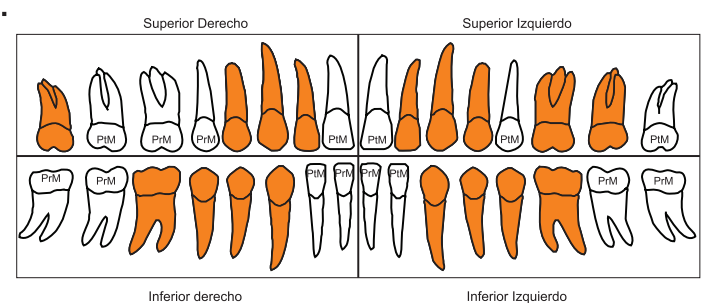

Figura 2. a. Representación de los elementos óseos presentes en el individuo recuperados en el sitio El Molino. En color naranja se indican los huesos presentes y en beige los fragmentados, mientras que el blanco señala la ausencia del hueso. b. Representación de la completitud dental. En naranja se indican las piezas dentales presentes y en blanco las ausentes (PrM: pérdida pre-mortem; PtM: pérdida post-mortem).

Figure 2. $\boldsymbol{a}$. Representation of the bone elements present in the individual recovered at El Molino. The present bones are indicated in orange and the fragmented ones in beige, while white indicates the absence of the bone. $\mathbf{b}$. Representation of dental completeness. Present teeth are indicated in orange and missing teeth in white (PrM: pre-mortem loss; PtM: postmortem loss).

discuten la posibilidad de que hayan sido consecuencia de la realización de actividades de molienda y de transporte de elementos pesados. El hecho de que el individuo de El Molino tenga una edad estimada alrededor de los 50 años, momento en el cual generalmente comienzan a aparecer alteraciones degenerativas en las articulaciones como producto del envejecimiento (Plischuk y Salceda, 2011; Weiss y Jurmain, 2007) podría concordar con esta hipótesis, y es esperable en el contexto de las prácticas agrícolas de las poblaciones tardías del área (Valencia, Flores, Wynveldt y Balesta, 2017).

El resultado de la datación radiocarbónica fue de $558 \pm$ 26 años AP (AA-111409). Los rangos de edad calibrada
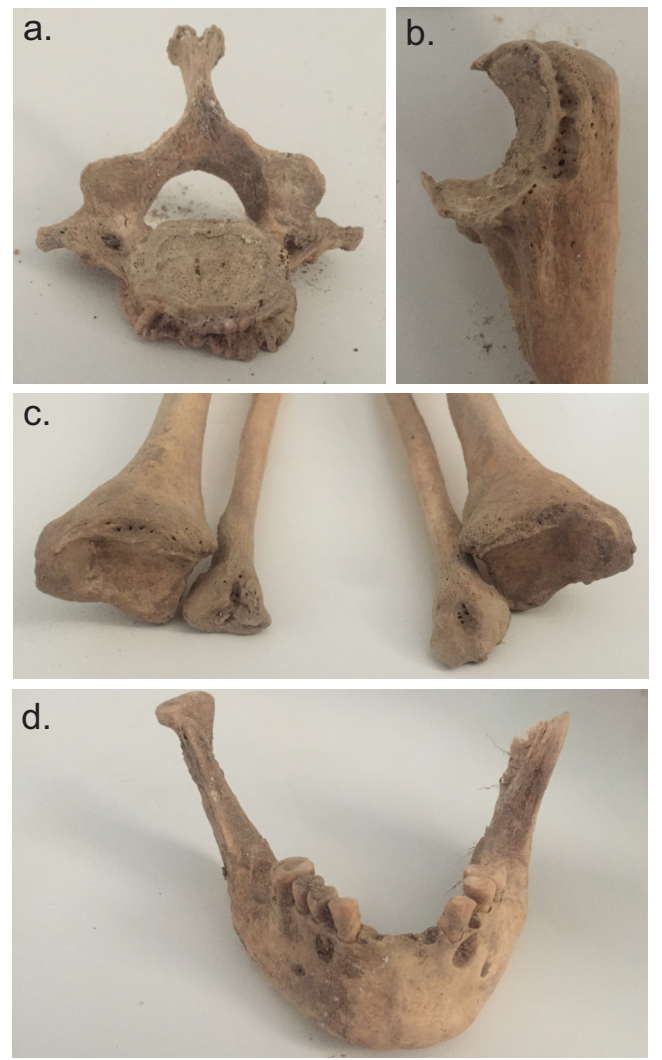

Figura 3. Imágenes de las patologías postcraneales y maxilares identificadas en el esqueleto del entierro. a. Vértebra cervical con osteofitos y reborde marginal en la superficie articular del cuerpo (osteoartrosis). b. Cúbito proximal derecho con características articulares degenerativas (osteoartrosis). c. Tibia y peroné derecho e izquierdo distales con características articulares degenerativas (osteoartrosis). d. Mandíbula con abscesos en el primer premolar derecho e izquierdo; desgaste dental en piezas dentarias mandibulares.

Figure 3. Postcranial and maxillary pathologies identified in the burial skeleton. $\boldsymbol{a}$. Cervical vertebra with osteophytes and marginal ridge on the articular surface of the body (osteoarthrosis). b. Right proximal ulna with degenerative joint features (osteoarthrosis). c. Distal right and left tibia and fibula with degenerative joint features (osteoarthrosis). $\boldsymbol{d}$. Mandible with abscesses in the right and left first premolar; dental wear in mandibular teeth.

son: $1407-1430$ d.C. $(1 \sigma, 68,2 \%$ prob.) y $1398-1442$ d.C. $(2 \sigma, 95,4 \%$ prob.). Este fechado es estadísticamente indiferenciable del obtenido del esqueleto de un niño del mismo sitio (García Mancuso e lucci 2008) y puede insertarse dentro de la propuesta cronológica de las ocupaciones Belén (Wynveldt, Balesta, lucci, Valencia y Lorenzo, 2017), que las ubica en su mayoría en el siglo XV.

Los resultados del análisis de los isótopos estables $\mathrm{C}^{13} \mathrm{y}$ $\mathrm{N}^{15}$, y su distribución en el espacio isotópico construido a partir de valores de humanos y de recursos previamente publicados para la región (Tablas Suplementarias 1 y 2), muestran que el individuo de El Molino tenía una dieta basada principalmente en plantas C4 (Plantas C4 B) y 


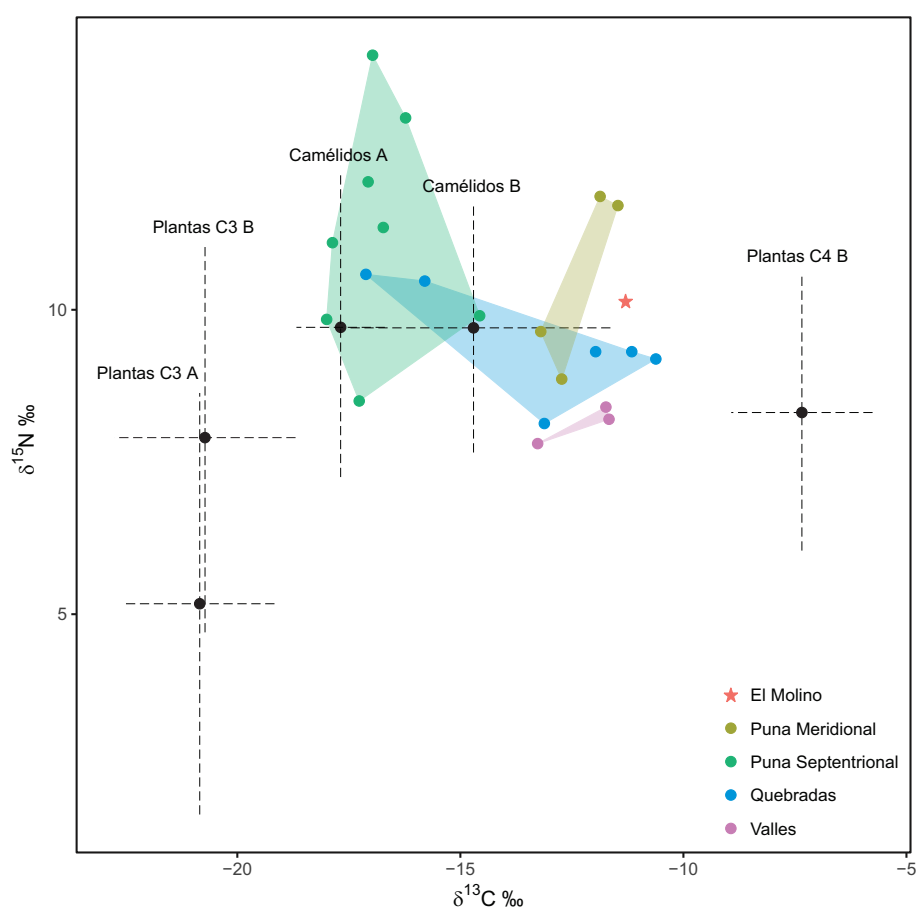

\section{Agradecimientos}

Agradecemos al Dr. Marcos Plischuk por sus observaciones sobre el diagnóstico de las paleopatologías. A su vez, a los dos evaluadores anónimos, cuyas sugerencias y comentarios contribuyeron a mejorar este trabajo.

\section{Referencias}

Balesta, B., y Zagorodny, N. (2000). Memorias e intimidades de una colección arqueológica. Relaciones de la Sociedad Argentina de Antropología, XXV, 41-50.

Balesta, B., y García Mancuso, R. (2010). Entierros infantiles en una habitación del cerro Colorado de la Ciénaga de Abajo. En Balesta, B. y Zagorodny N. (Eds.), Aldeas protegidas, conflicto y abandono. Investigaciones Arqueológicas en La Ciénaga (Catamarca, Argentina) (pp. 241275). Al Margen.
Figura 4. Espacio isotópico de $\delta 13 \mathrm{C}$ y $\delta 15 \mathrm{~N}$ para los recursos y los humanos, agrupados por altitud $(A=$ alta y $B=$ baja) y por región de origen, respectivamente.

Figure 4. Isotopic space of $\delta 13 C$ and $\delta 15 N$ for resources and humans, grouped by altitude $(A=$ high and $B=$ low) and by region of origin, respectively.

camélidos de regiones bajas (Camélidos B; Figura 4). Resultados similares se observan en otros individuos provenientes de Valles, Quebradas y Puna Meridional del Noroeste Argentino. Asimismo, este resultado es coherente con la altura a la que se encuentra el sitio (1.932 msnm), así como con los indicadores de desgaste y patologías dentales que sugieren la ingesta de altas proporciones de recursos vegetales procesados (probablemente maíz).

\section{Conclusión}

Este trabajo constituye el primer estudio sistemático de las características biológicas de un entierro humano adulto en el sitio El Molino. El mismo presenta evidencia consistente con una dieta basada preferentemente en recursos agrícolas y rastros de patologías frecuentes en grupos humanos agricultores. Futuros estudios, que incluyan un número mayor de casos del mismo sitio o del área, posibilitarán conocer en qué medida estas características eran frecuentes en la población durante la última etapa de desarrollo histórico prehispánico.

La Plata, 29 de abril de 2020
Dantas, M., Figueroa, G., Laguens, A., e Izeta, A. (2014). Isótopos estables, dieta de camélidos y diferenciación social (Valle de Ambato, Catamarca, Argentina, siglos VI-XI d.C.). Revista Chilena de Antropología, 30, 90-97.

Dembo, A., e Imbelloni, J. (1938). Deformaciones intencionales del cuerpo humano de carácter étnico (Vol. 3). Editorial Nova.

García Mancuso, R. e lucci, M. E. (2008). Entierro infantil en un contexto doméstico (El Molino, Puerta de Corral Quemado, Catamarca). Resúmenes del X Congreso de la Asociación Latinoamericana de Antropología Biológica.

González, A. R. (1955). Contextos culturales y cronología relativa en el área central del N. O. argentino (nota preliminar). Anales de Arqueología y Etnología, 9, 7-32.

González, A. R., y Cowgill, G. L. (1975). Cronología arqueológica del Valle de Hualfín, Pcia. de Catamarca, Argentina. Obtenida mediante el uso de computadoras. Actas del Primer Congreso de Arqueología Argentina, 383-395.

Gordón, F., Perez, S. I., Hajduk, A., Lezcano, M., y Bernal, V. (2018). Dietary patterns in human populations from northwest Patagonia during Holocene: an approach using Binford's frames of reference and Bayesian isotope mixing models. Archaeological and Anthropological Sciences, 10, 1347-1358.

Grant, J. (2016). Isótopos estables en camélidos y vegetales modernos de Antofagasta de la Sierra: hacia una ecología isotópica de la Puna Meridional Argentina. Intersecciones en Antropología, 17(3), 327-339. 
Killian Galván, V. S., y Samec, C. T. (2012). A cada uno su verdad culinaria: Patrones paleodietarios y variables ambientales en el NOA. En N. Kuperszmit, T. Lagos Mármol, L. Mucciolo, y M. Sacchi (Eds.), Entre pasados y presentes III. Estudios contemporáneos en ciencias antropológicas (pp. 487-508). Mnemosyne.

Killian Galván, V. A., Sanmartino, G., Castellano, V., Seldes, V., y Marban, L. G. (2015). Estudios de isótopos estables en huertas familiares actuales de la Quebrada de Humahuaca (Jujuy, Argentina). Su potencial aporte a los estudios paleodietarios del Noroeste argentino. Revista del Museo de Antropología, 8(2), 107-118.

Lamenza, G. N., Desántolo, B., Drube, H., Calandra, H., Salceda, S., y Sempé, C. (2016). Nuevos aportes a la arqueología del Valle de Hualfín: el sitio Cardón Mocho de Azampay (Belén, Catamarca). Revista Española de Antropología Americana, 46, 305-328.

Larsen, C. S. (1987). Bioarchaeological Interpretations of Subsistence Economy and Behavior from Human Skeletal Remains. En Schiffer, M. B. (Ed.), Advances in Archaeological Method and Theory, vol. 10, (pp. 339-445), Academic Press.

Lyman, R. L. (1994). Vertebrate taphonomy. Cambridge University Press.

Mengoni Goñalons, G. L. (1999). Cazadores de guanacos de la estepa patagónica. Sociedad Argentina de Antropología.

Olivera, D. E., y Yacobaccio, H. D. (1999). Estudios de paleodieta en poblaciones humanas de los Andes del Sur a través de isótopos estables. Trabajo presentado al V Congreso Nacional de Paleopatología, Alcalá La Real, Jaén.

Onaha, M. E., Tobisch, A., Padula, G., Drube, H., y Vasallo, M. (2002). Entierro humano en la Mesada de Carrizal, Valle de Hualfín (Dto. Belén, Pcia. De Catamarca). En Berberián, E. (Ed.), Actas del XIII Congreso Nacional de Arqueología Argentina (pp. 497-510).

Palamarczuk, V., y Ratto, N. R. (2015). Un encuentro de arqueologías: rescates y estudio de colecciones del inicio del Período Tardío (ca. Siglos XI-XIII) (Catamarca, Argentina). Revista del Museo de Antropología de Córdoba, 8, 91-106.

Plischuk, M., y Salceda, S. (2011). Evidencia esquelética de osteoartritis en una muestra contemporánea. Revista Española de Antropología Física, 32, 43-49.

Samec, C. T., Yacobaccio, H. D., y Panarello, H. O. (2017). Carbon and Nitrogen Isotope Composition of Natural Pastures in the Dry Puna of Argentina: A Baseline for the Study of Prehistoric Herd Management Strategies. Archaeological and Anthropological Sciences, 9(2), 153-163.

Samec, C. T., Pirola, M., Yacobaccio, H. D., y Panarello,
H. O. (2018). Assessing Prehispanic Herding Strategies through Stable Isotope Analysis: A Case Study from the Dry Puna of Argentina. Environmental Archaeology, 1-12. https://doi.org/10.1080/14614103.2018.1549348.

Scott, E. (1979). Dental wear scoring technique. American Journal of Physical Anthropology, 51, 213-217.

Sempé, M. C. (1981). Investigaciones arqueológicas en el departamento Belén (Catamarca). Novedades del Museo de La Plata, 1(2), 18-19.

Sempé, M. C., Salceda, S., y Maffia, M. (2005). Azampay. Presente y pasado de un pueblito catamarqueño. Ediciones Al Margen.

Sempé, M. C., Méndez, G., y Salceda, S. (1995-1996). Entierro intrusivo en Barrealito de Azampay. Shincal, 5, 43-49.

Smith, B. H. (1984). Patterns of molar wear in huntergatherers and agriculturalists. American Journal of Physical Anthropology, 63, 39-56.

Stock, B. C., y Semmens, B. X. (2016). MixSIAR GUI User Manual. Version 3.1. https://github.com/brianstock/ MixSIAR. doi:10.5281/zenodo.1209993.

Ubelaker, D. (2008) Human Skeletal Remains: Excavation, Analysis, Interpretation. Aldine Transaction.

Valencia, C., Flores, M., Wynveldt, F., y Balesta, B. (2017). Identificación de variedades de maíz y prácticas agrícolas en el valle de Hualfín (Catamarca, Argentina). Revista Española de Antropología Americana, 46, 283-304. https://doi.org/10.5209/REAA.58298.

Weiss, E., y Jurmain, R. (2007) Osteoarthritis Revisited: A Contemporary Review of Aetiology. International Journal of Osteoarchaeology, 17, 437-450.

Weisser, W. (1924). Diarios de viaje pertenecientes a la Colección Muñiz Barreto. Departamento Arqueología del Museo de La Plata, La Plata.

White, T. D., y Folkens, P. A. (2005). The human bone manual. Elsevier.

Wynveldt, F. (2009). La loma de los Antiguos de Azampay. Un sitio defensivo del valle de Hualfín (Catamarca, Argentina). Sociedad Argentina de Antropología.

Wynveldt, F., Balesta, B., lucci, M. E., Valencia, C., y Lorenzo, G. (2017). Late chronology in Hualfin valley (Catamarca, Argentina): a revisión from ${ }^{14} \mathrm{C}$ dating. Radiocarbon, 59(1), 91-107. https://doi.org/10.1017/RDC.2016.114.

Wynveldt, F., e lucci, M. E. (2013). La cronología Belén en el norte del valle de Hualfín: viejos problemas, nuevas perspectivas. Arqueología, 19(1), 187-195. 